\title{
MAGIS-100 Atom Source Support Preliminary Design
}

\author{
Kelsey Scheidt, CCI \\ Mentors: Linda Valerio, Jesse Batko, Beth Klein
}

\section{Atom Source overview}

There are three Atom Sources within the MAGIS-100 experiment, located at the ends and midpoint of the shaft, adjacent to the vacuum tube.

Each Atom Source weighs approximately 700 pounds and contains the optical components for the atom interferometry and delivers the Strontium.

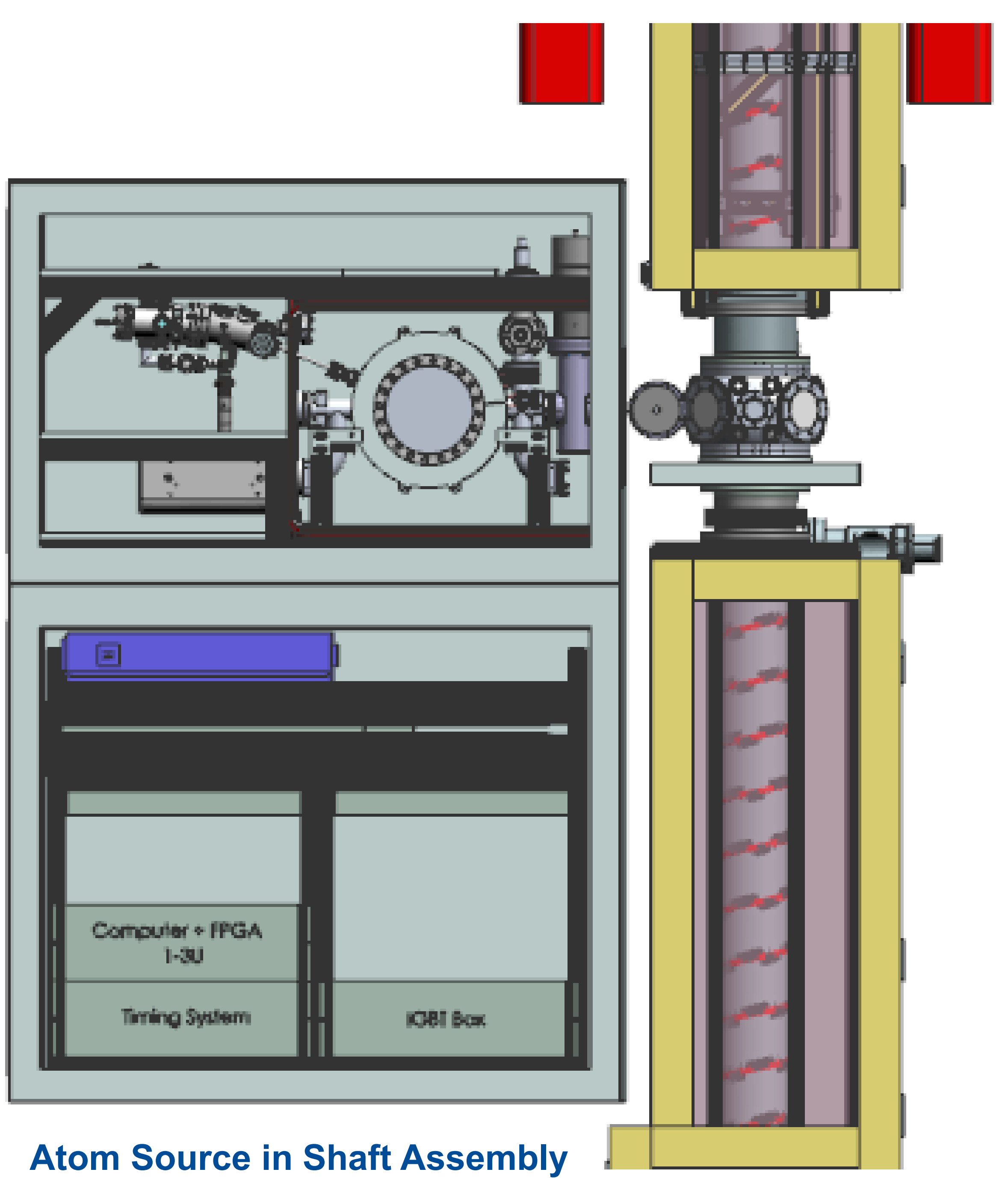

\section{Process}

My process for the design followed three main steps: Initial hand sketches were completed to lay out the possible designs of each component, beginning with the main support structure.

NX models were then made to visualize the structure in three dimensions, allowing for easier redesigns.

Finally, NASTRAN simulations were done, and the model was adjusted from the data received.

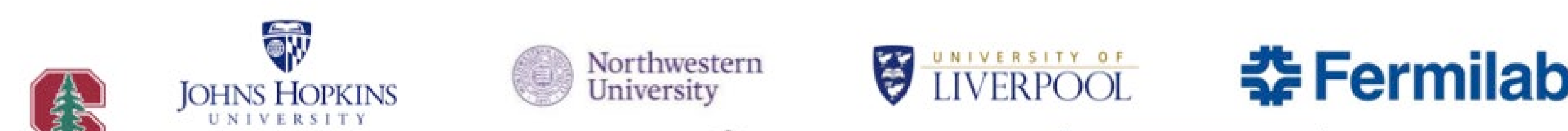

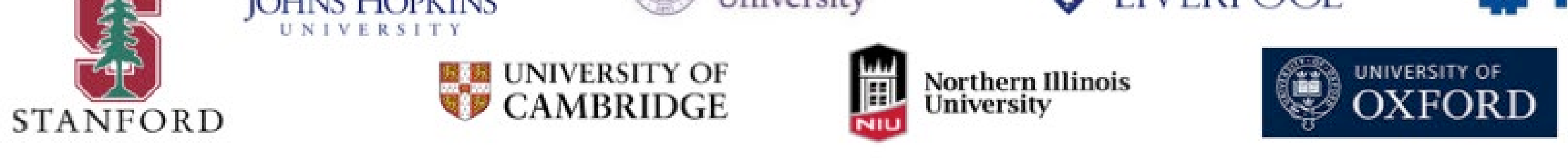

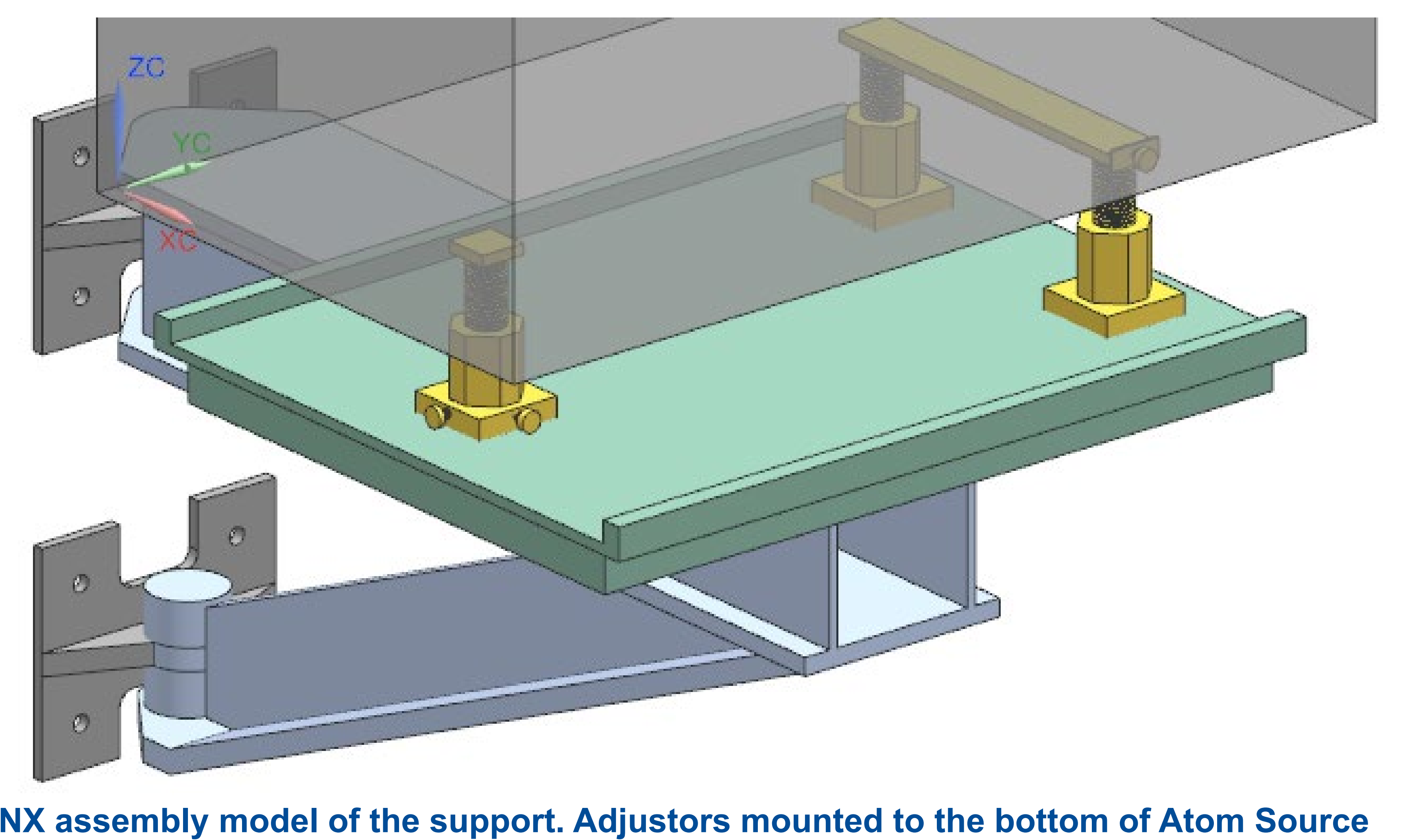
$\mathrm{NX}$ ass
frame.

The supports are made of three main components:

Main Support Structure: Made of two flat plates, 47 inches in length, ending in rounded triangles to allow for pivoting.

Wall Attachment Plate: 12×10 inch plate with triangular attachment points and space for the support to rotate through it. Specifics of securing the plate will be analyzed by FESS civil engineers.

Adjusters and Adjuster Plate: 3-point adjuster design based off existing Fermilab designs. Adjuster plate design is not final.

Additional designs for a pivoting system were made and accounted for, but the completion of which was outside the scope of this internship.

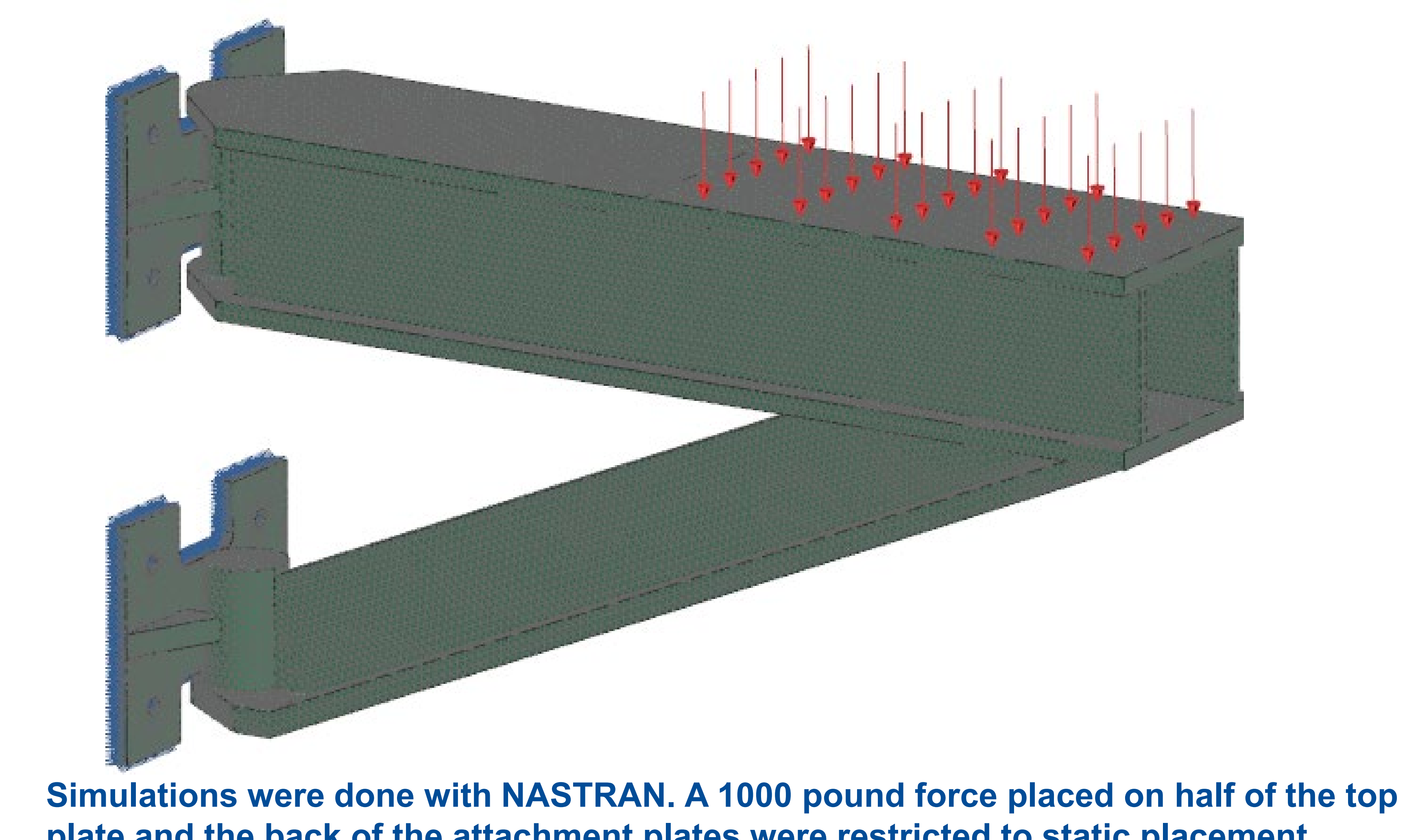
plate and the back of the attach

\section{Simulation Results}

The stress and displacement of the structure were simulated with NASTRAN. All components were set to be made of 304 Stainless Steel (also known as 18/8), however 316 Stainless Steel would also be acceptable for this application for their shared corrosion resistance and non-magnetic properties.

A 1000-pound force was placed on the top plate of the Main Support Structure in the area which overlapped with the Adjuster Plate. Additionally, the back face of the Attachment Plates was secured to remain static as they would be when installed.

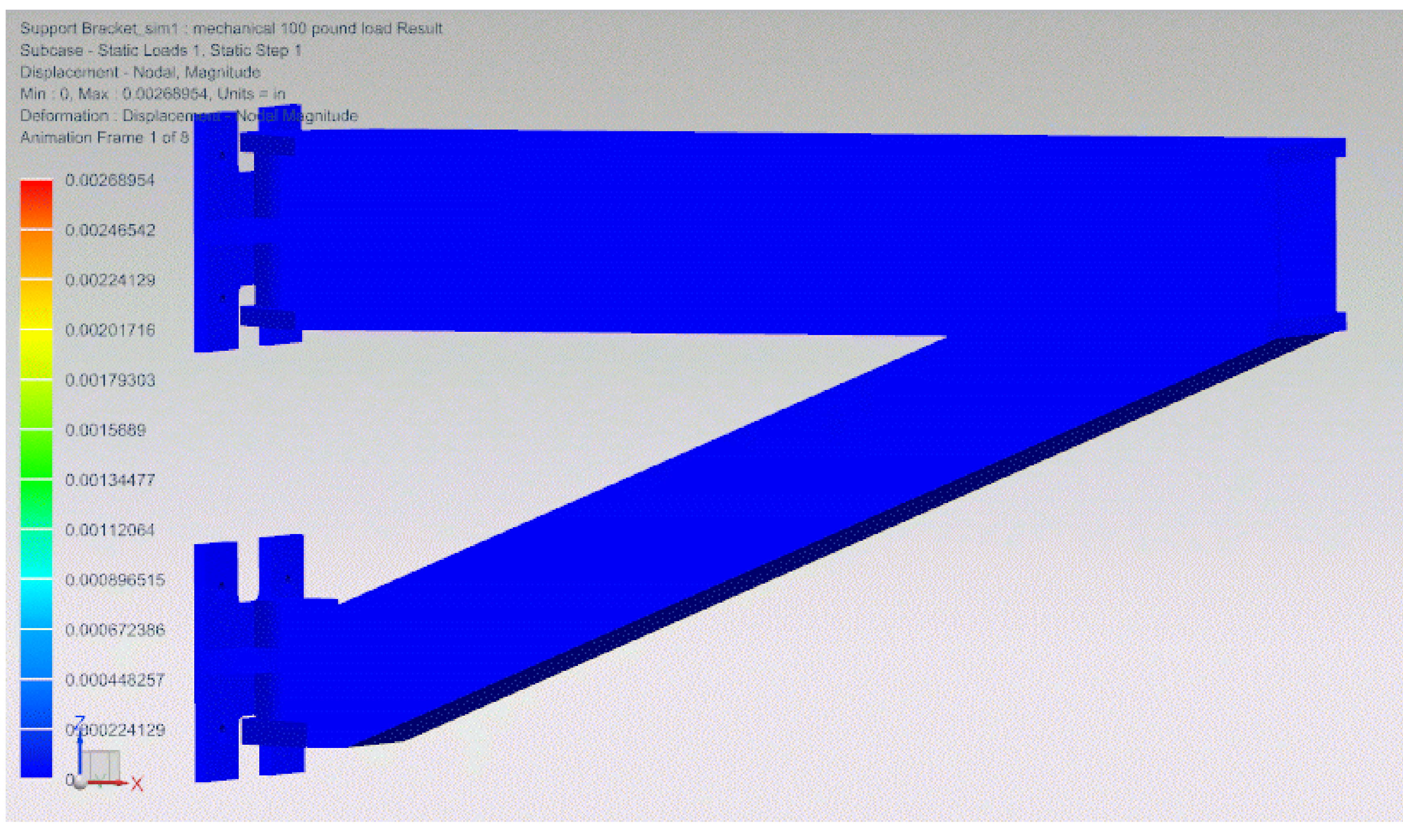

Displacement of the Main Support Structure and Attachment Plate. Maximum Displacement: 0.00268954 in

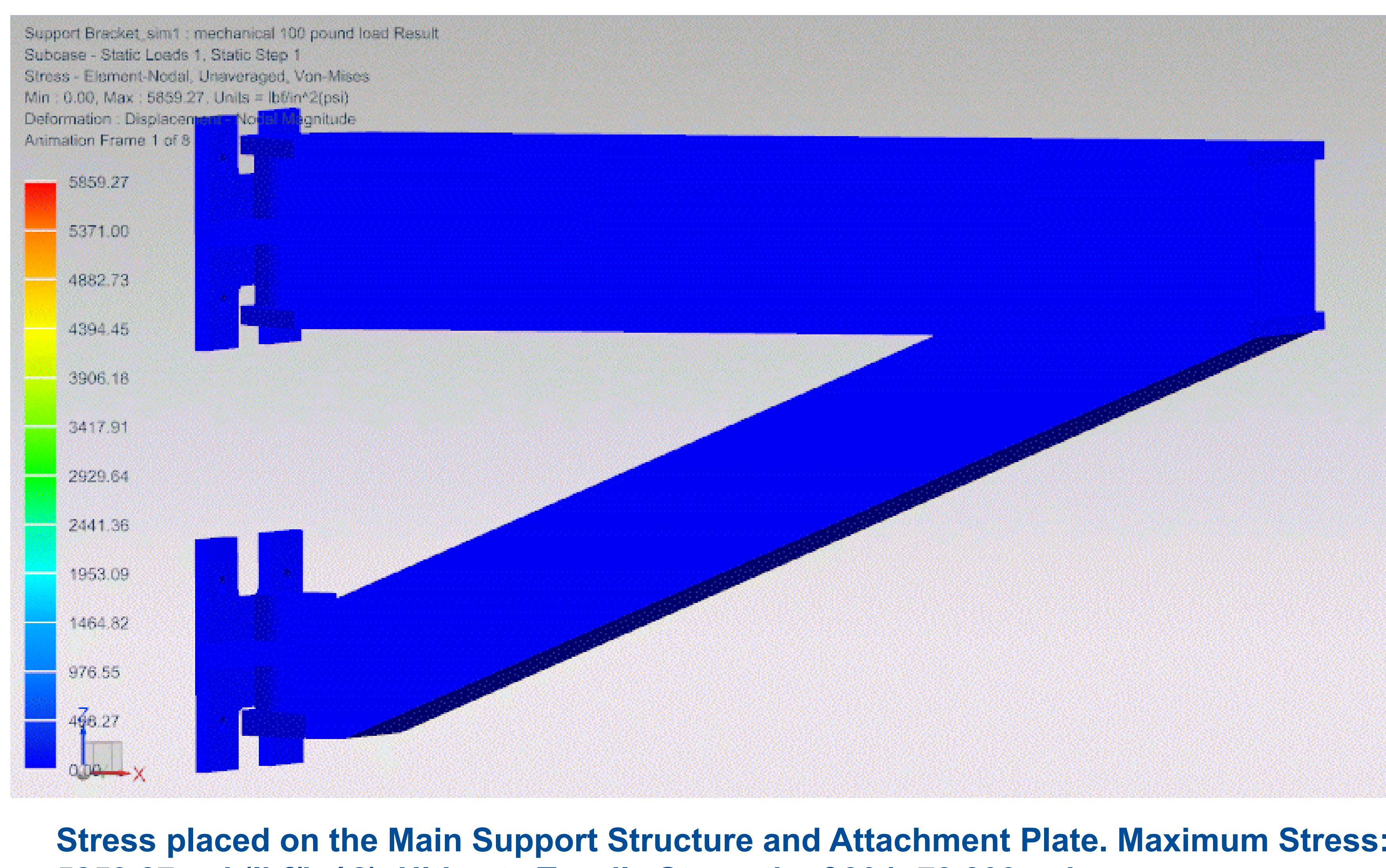
Stress placed on the Main Support Structure and Attachment Plate.
5859.27 psi (lbf/in^2). Ultimate Tensile Strength of 304: 73,200 psi 